\title{
COMPUTED TOMOGRAPHY AND MAGNETIC RESONANCE IMAGING FINDINGS IN A RARE CASE OF COMMON CAVITY MALFORMATION- A CASE REPORT
}

Yasmin $S^{1}$, Javaji Ravi Prasad², Hazif Backer P3, Parthasarathi $A^{4}$

${ }_{1}^{1}$ Postgraduate Student, Department of Radiodiagnosis, Rajarajeswari Medical College and Hospital, Bengaluru.

2 Professor, Department of Radiodiagnosis, Rajarajeswari Medical College and Hospital, Bengaluru.

${ }_{3}^{3}$ Postgraduate Student, Department of Radiodiagnosis, Rajarajeswari Medical College and Hospital, Bengaluru.

${ }^{4}$ Assistant Professor, Department of Radiodiagnosis, Rajarajeswari Medical College and Hospital, Bengaluru.

HOW TO CITE THIS ARTICLE: Yasmin S, Prasad JR, Backer HP, et al. Computed tomography and magnetic resonance imaging findings in a rare case of common cavity malformation- a case report. J. Evolution Med. Dent. Sci. 2017;6(37):3048-3050, DOI: $10.14260 /$ Jemds/2017/657

\section{PRESENTATION OF CASE}

A 40-year-old female patient presented to our hospital with complaints of hearing loss since birth. There was complete hearing loss on the right side. On the left side, there was very mild hearing loss. She was unable to tolerate loud sounds and had multiple episodes of tinnitus. She also complained of episodes of dizziness and nausea. There was no family history of similar complaints.

On examination, the pinnae and the external auditory canals were normal on both sides. Bilateral tympanic membranes were intact. The patient underwent a tympanometry examination. It showed type A subtype tympanogram on the right side. This is indicative of mild ossicular chain stiffness. Mild low-frequency hearing loss was seen on the left side.

The patient was referred to the Radiodiagnosis Department for an MRI scan. MRI scan revealed an inner ear abnormality on the right side. It showed the confluence of vestibule and cochlea into a single cystic cavity on the right side (Figure 1 and 2). The cystic cavity had a vertical diameter of $7 \mathrm{~mm}$ and a horizontal diameter of $11 \mathrm{~mm}$. The semicircular canals were also deformed on the same side (Figure 3). Right middle ear and external ear cavities were normal. The left external and middle ear cavities were also normal. Normally developed and differentiated cochlea, vestibule and semicircular canals were seen on the left side. The cerebrum, cerebellum and brainstem were also normal.

The patient also underwent an HRCT temporal bone to better assess the middle ear cavity. It showed the left cochlea and vestibule being replaced by a common cavity as seen on MRI (Figure 4 and Figure 5). The external ear cavities and tympanic membranes were normal on both sides. It also showed bilaterally normal middle ear cavities with normal ear ossicles. Left vestibule, cochlea and semi-circular canals were also normal. The mastoids were normal on both sides and showed normal pneumatisation. Facial nerves were normal bilaterally.

Financial or Other, Competing Interest: None.

Submission 30-03-2017, Peer Review 24-04-2017,

Acceptance 29-04-2017, Published 08-05-2017.

Corresponding Author:

Dr. Hazif Backer $P$,

Room No. 134, Male Residents Hostel,

Rajarajeswari Medical College and Hospital,

Kambipura, Mysore Road,

Bengaluru-560074.

E-mail: hazifbacker@gmail.com

DOI: $10.14260 /$ jemds $/ 2017 / 657$

(c) (i) $\$$

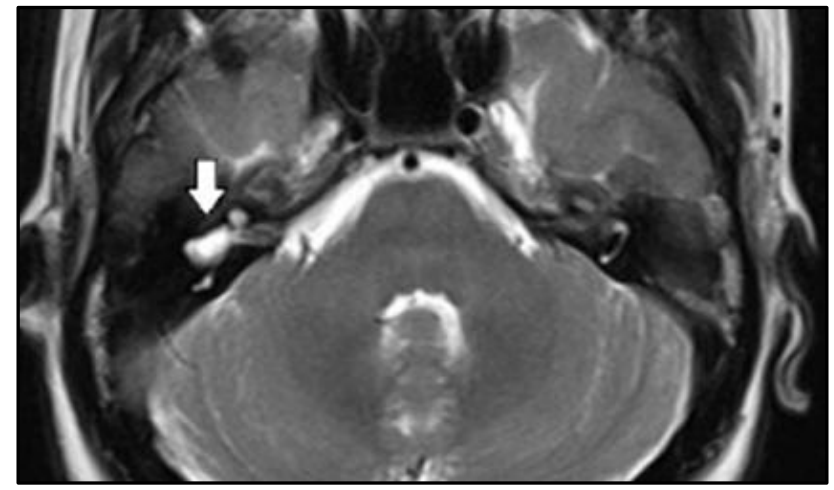

Figure 1. Axial T2 Weighted MR Image of the Inner Ear showing the Common Cavity Malformation on the Right Side (Arrow)

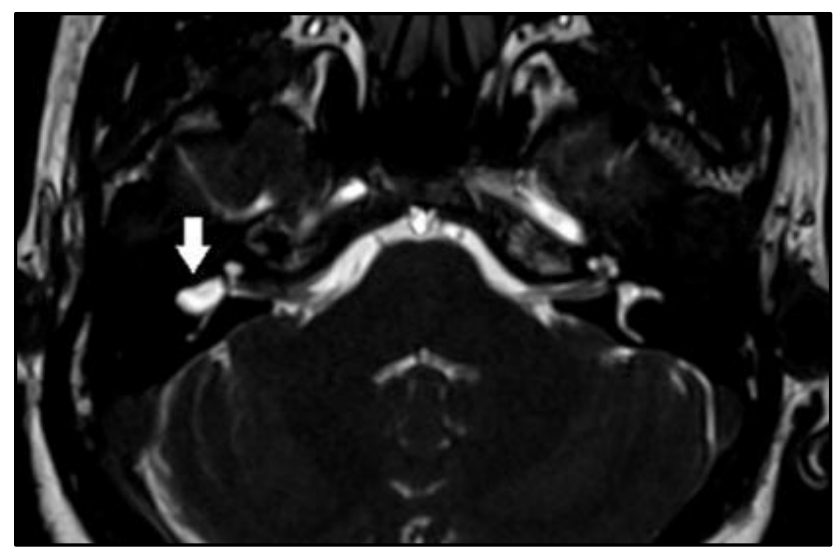

Figure 2. Axial 3D CISS MR Image of the Inner Ear showing a Single Cystic Structure replacing the Cochlea and Vestibule on the Right Side (Arrow)

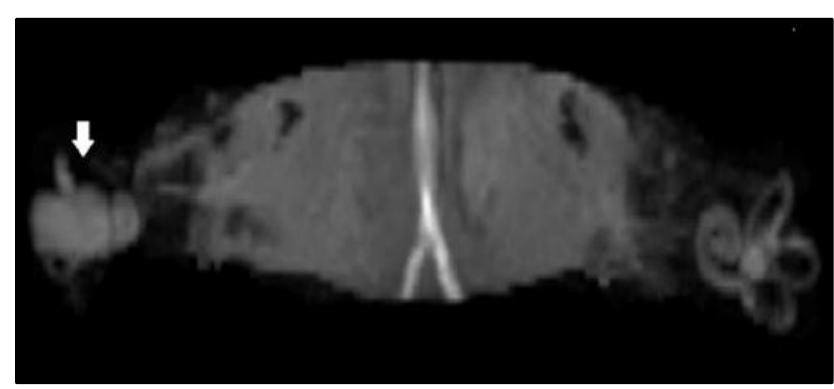

Figure 3. 3D CISS Maximum Intensity Projection (MIP) MR Image of the Inner Ear showing the Deformed Semicircular Canals and Common Cavity Malformation on the Right Side(Arrow) 


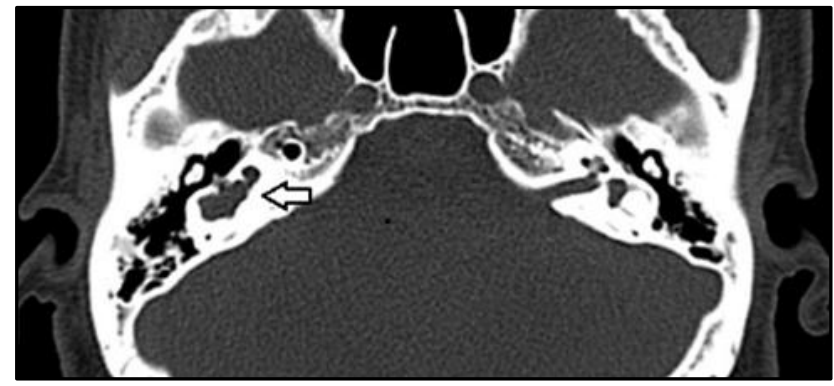

Figure 4. Axial High-Resolution CT Scan of the Temporal Bone showing the Right Cochlea and Vestibule being replaced by a Common Cystic Cavity (Arrow)

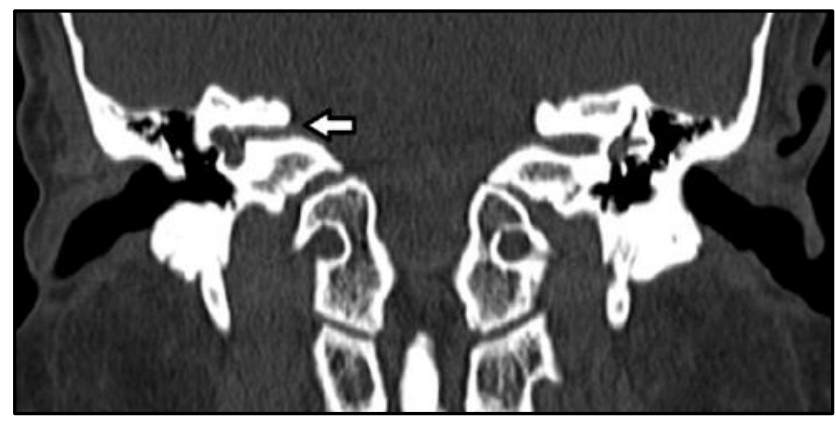

Figure 5. Coronal High-Resolution CT Scan of the Temporal Bone showing the Common Cavity Malformation on the Right Side (Arrow) with Normal Inner Ear Cavity on the Left Side

\section{DISCUSSION}

Cochlear malformations are a class of congenital inner ear anomalies that leads to congenital sensorineural deafness. It is an important cause of sensorineural hearing loss in children. These malformations can also vary in their severity. They can be mild dysplasias or total aplasia of the inner ear. The cochlea is ectodermal in origin arising from the otic placode and otic vesicle. Cochlear development begins from the third week of intrauterine gestation and is completed by the seventh week. Based on the week at which developmental arrest occurs different types of cochlear anomalies manifest. This insult during embryogenesis may be due to a variety of factors. They may be either environmental or genetic.(1)

Jackler et al(2) first came up with a classification of cochlear developmental anomalies based on the week at which development arrest occurs. This was later modified by Sennaroglu et $\mathrm{al}^{(3)}$ in 2002. They classified them into six categories based on the timing of insult. They comprise of complete labyrinthine aplasia also called as Michel deformity (third week), cochlear aplasia (late third week), common cavity malformation of the cochlea and vestibule (fourth week), type I incomplete partition (fifth week), cochlear hypoplasia (sixth week) and incomplete partition type II (seventh week).

Common cavity malformation or deformity was first explained by Cock in 1838.(4) It is due to developmental arrest at the fourth week of intrauterine development. It accounts for one-fourths of all cochlear malformations. Here, the vestibule and cochlea fail to differentiate into two different anatomical structures due to developmental insult. They are replaced by a single common cystic structure in the inner ear. This leads to sensorineural hearing loss in the patient. The common cavity is wider than tall with an average height of $7 \mathrm{~mm}$ and width of $10 \mathrm{~mm}$.(5) It can also be associated with deformities in the semi-circular canals as seen in our patient. Ossicles can be normal as seen in our patient or the stapes can be deformed. Abnormal path of the facial nerve as well as abnormalities of the vestibulocochlear nerves can also be a feature of this malformation. There is also a failure of the vestibular aqueduct to dilate in some cases.

High-resolution CT scan of temporal bone plays an important role in diagnosis of common cavity malformation. Ear ossicles as well as other bony structures can be well assessed by this study and it also helps in ruling out other causes of deafness like conductive deafness. HRCT scan also helps in studying the external ear cavity.(6) Inner ear structures like the cochlea, vestibule and semicircular canals can also be seen using HRCT scan. Thus, it plays an indispensable role in arriving at a diagnosis, which can explain the deafness in the patient.

MRI helps in assessing the inner ear and the cranial nerves like facial and vestibule-cochlear nerves. T2 weighted images are better for assessing the inner ear. In T2 weighted images, the common cavity malformation presents as an hyperintense fluid filled cyst in the inner ear cavity with deformed ossicles. 3D CISS MR sequence also helps in assessing the semicircular canals and cochlea well. 3D CISS Maximum Intensity Projection (MIP) MR image helps in demonstrating the cochlea and semicircular canals in great detail. HRCT temporal bone along with MRI helped in arriving at a conclusive diagnosis of right-sided common cavity deformity in our patient.

\section{DISCUSSION OF MANAGEMENT}

Identifying and correctly diagnosing the type of congenital inner abnormality is of utmost importance in the planning of suitable management. Common cavity malformation can be successfully treated by cochlear implantation.(7) However, post-surgical results are less favourable compared to children with intact inner ear. Cerebrospinal fluid leak can be associated with common cavity malformation. This should be anticipated and corrected while performing cochlear implantation.

\section{FINAL DIAGNOSIS}

Common cavity malformation is a congenital cochlear malformation resulting from a developmental arrest of the cochlea during the fourth week of intrauterine life. HRCT temporal bone and MRI plays an important role in diagnosing this malformation. Prompt diagnosis using these modalities helps in the early treatment of these patients by cochlear implantation.

\section{REFERENCES}

[1] Yiin RS, Tang PH, Tan TY. Review of congenital inner ear abnormalities on CT temporal bone. $\mathrm{Br} \mathrm{J}$ Radiol 2011;84(1005):859-63.

[2] Jackler RK, Luxford WM, House WF. Congenital malformations of the inner ear: a classification based on embryogenesis. Laryngoscope 1987;97(3 Pt 2 Suppl 40):2-14. 
[3] Sennaroglu L, Saatci I. A new classifcation for cochleovestibular malformations. Laryngoscope 2002;112(12):2230-41.

[4] Schmidt RS, Sismanis A. Common cavity deformity. Otology \& Neurotology 2008;29(4):567-8.

[5] Joshi VM, Navlekar SK, Kishore GR, et al. CT and MR imaging of the inner ear and brain in children with congenital sensorineural hearing loss. Radiographics 2012;32(3):683-98.
[6] Casselman JW, Offeciers EF, De Foer B, et al. CT and MR imaging of congential abnormalities of the inner ear and internal auditory canal. Eur J Radiol 2001;40(2):94-104.

[7] Buchman CA, Copeland BJ, Yu KK, et al. Cochlear implantation in children with congenital inner ear malformations. Laryngoscope 2004;114(2):309-16. 